\title{
Matières à friction et techniques de lutte
}

Introduction

\section{Matthieu Duperrex et Mikaëla Le Meur}

\section{CpenEdition}

Journals

Édition électronique

URL : https://journals.openedition.org/tc/14132

DOI : $10.4000 /$ tc. 14132

ISSN : 1952-420X

Éditeur

Éditions de l'EHESS

\section{Édition imprimée}

Date de publication : 30 octobre 2020

Pagination : 12-25

ISBN : 978-2-7132-2840-7

ISSN : 0248-6016

Référence électronique

Matthieu Duperrex et Mikaëla Le Meur, « Matières à friction et techniques de lutte », Techniques \& Culture [En ligne], 74 | 2020, mis en ligne le 28 octobre 2020, consulté le 04 janvier 2023. URL : http:// journals.openedition.org/tc/14132; DOI : https://doi.org/10.4000/tc.14132 


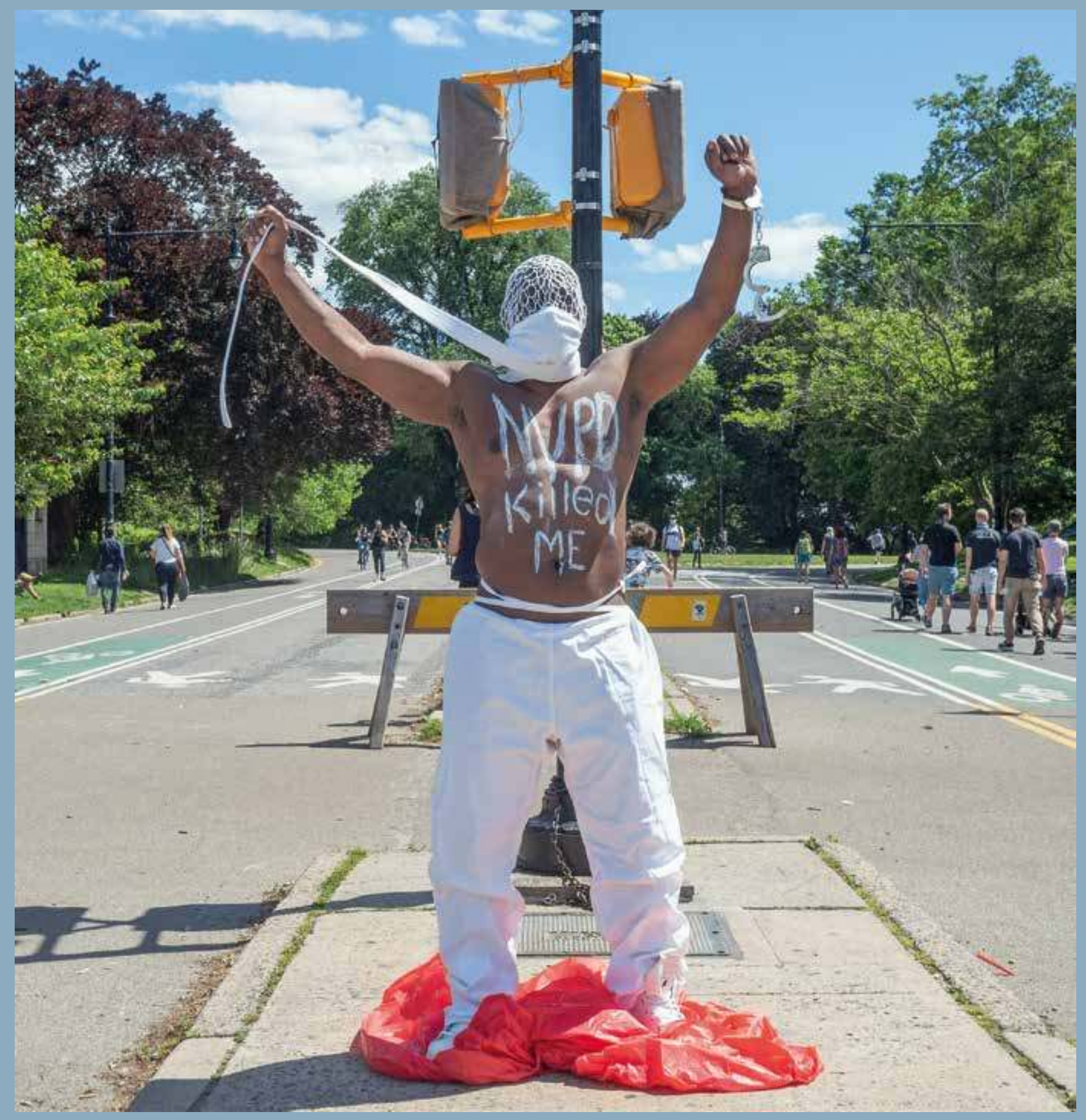




\section{Matières à friction et techniques de lutte}

On étouffe. La situation n'est pas tenable. Nous courons à la catastrophe. L'effet de sidération paralyse les velléités d'action. Aussi diverses et parfois contraires que soient les causes et les situations, ce contre quoi nous avons des raisons de nous insurger semble se fondre dans un même mouvement global, une lame de fond irrépressible.

«Nous sommes plus nombreux, incomparablement plus nombreux. Il y a maintenant un ton de désespoir dans nos voix; nous parlons d'extinction et d'avenir annulé. Et pourtant, les affaires continuent tout à fait comme avant - business as usual. À quel moment nous déciderons-nous à passer au stade supérieur? Quand conclurons-nous que le temps est venu d'essayer autre chose?» (Malm 2020: 11)

Bien sûr, l'appel à «passer au stade supérieur» dont le géographe activiste Andreas Malm se fait ici l'écho dans Comment saboter un pipeline n'est pas nouveau dans l'histoire des luttes et n'est d'ailleurs pas forcément de réjouissante mémoire. Bien sûr aussi, dans nos engagements et mobilisations, il nous faut apprendre sans cesse à trier et à distinguer clairement les points de friction et les sujets de contention dont la diplomatie n'a pu épuiser le motif. Toutefois, il nous semble d'un intérêt majeur que, régulièrement, la question de l'efficacité des moyens d'action se pose, outre celle de leur légitimité. Or la grande majorité des récits qui sont produits sur le vaste ensemble des «mouvements sociaux» s'attarde peu sur la matérialité des expériences, des modes opératoires ou encore sur les «techniques» de lutte. Quels instruments possédons-nous pour semer le trouble dans la mécanique des rapports de domination? Car, par gros temps, nous nous équipons toujours, moralement, matériellement. Les individus ou les collectifs qui résistent et opposent aux gouvernementalités de nouvelles priorités, d'autres perspectives, travaillent forcément leurs outils autant que leurs convictions. Ils suspendent le temps et gagnent du terrain par adaptation ou détournement de choses, ou en mettant hors d'usage des dispositifs, comme l'écologiste islandaise dans le film Woman at War (2018), experte dans l'art de saboter, non pas 
un pipeline, mais une ligne à haute tension ravitaillant une usine de fabrication d'aluminium. Les agencements matériels et techniques propres aux mouvements contestataires méritent que nous nous y arrêtions, que nous y consacrions a minima un temps de description sans neutraliser les dimensions organique et politique des luttes, c'est-à-dire en préservant leur caractère sensible.

\section{De l'expérience sensible au trouble}

Au commencement, c'est le cœur qui se soulève. Ce sont ses spasmes et ses humeurs qui nous travaillent et transforment le dégoût en sentiment d'injustice, la rage en colère, l'écœurement en indignation morale et politique. Au bord des lèvres, avant les mots peut-être, l'expression commune du soulèvement, c'est l'émeute. Elle partage avec l'émotion son étymologie médiévale: esmeu a donné «ému», «émouvoir ». L'émeute, cet émoi collectif ou «tumulte séditieux» provoqué par des corps agités et des esprits rassemblés, sème le trouble en son propre sein, comme au sein du pouvoir qui n'y voit qu'une secousse, un symptôme sans affection profonde:

«Le marquis de Botta crut que cette émeute du peuple [de Gênes] se ralentirait d'elle-même, et que la crainte reprendrait bientôt la place de cette fureur passagère. » Voltaire, Louis XV, 21 (Dictionnaire Littré.)

Le terme «émeute» fait pourtant la part belle à l'«animal politique» selon l'expression d'Aristote, un être de relation, de raison et de passion mêlées. De l'émeute à la révolte, des cris de ralliement aux cahiers de doléances, le partage des émotions favorise les mobilisations politiques et «l'enrôlement des soutiens» à une cause (Traïni \& Siméant 2009). Kay Milton montre ainsi comment des écologistes amorcent leurs carrières militantes après avoir vécu un « déclic», une expérience émotionnelle forte révélant leur «amour de la nature (Milton 2002). L'historienne Michelle Perrot, ramenant l'analyse des mouvements sociaux à l'intimité des expériences physiques et émotionnelles, considère quant à elle que l'«idée de grève générale sourd de l'expérience même des "bras croisés" où les producteurs éprouvent leur puissance. » (Perrot 2001: 7)

Cette imbrication entre expérience politique et expérience esthétique (dans son acception sensorielle) est contenue dans le terme "sensible», dont le partage est un enjeu pour les luttes et un chemin pour l'émancipation (Rancière 2000). Frontalier de ces deux univers a priori disjoints, ce terme «sensible» est assigné aux quartiers de banlieue, lieux bannis par la Cité et au bord de l'embrasement permanent, qui ont été, durant l'hiver 2005, le théâtre d'«émeutes» dont les destructions matérielles - voitures brûlées, vitres de panneaux publicitaires ou d'abris de bus brisées, etc. - ont marqué l'imaginaire des luttes urbaines françaises. Il permet également de qualifier le regard de Louise Michel sur le monde, à l'examen de ses cahiers de bagne foisonnants d'esquisses paysagères et de détails végétaux de Nouvelle-Calédonie, elle qui fut à la fois figure de la Commune de Paris (18 mars-28 mai 1871), poétesse et dessinatrice, luttant 
ardemment pour que les femmes soient considérées à l'égal des hommes, dans la cité comme en prison (Tchernookov 2018).

Cette sensibilité esthétique et politique à la vitalité et à la matérialité du monde, au devenir commun des humains et des non-humains - femmes, hommes, «mobilier urbain », pierres ou végétaux - dans le contexte actuel caractérisé par des bouleversements écologiques majeurs et des «violences natureculturelles sans précédent», fait dire à Donna Haraway, à la suite de Judith Butler (Butler 2006) qu'il faut «habiter le trouble» (Caeymaex et al. 2019). Cette proposition philosophique et politique soucieuse de reconstruire du lien ou «de la parenté en chaîne» (Haraway 2020) fait écho aux travaux proposant de rematérialiser le politique, trop souvent cantonné à la sphère des idées (Braun \& Whatmore 2010). Les matières à questions et les choses publiques (res publica) sont autant des problèmes conceptualisés que des choses concrètes et tangibles. Il en va ainsi de l'offensive des poubelles au sein d'un barrio new-yorkais dégradé, que décrit Claire Richard dans ce numéro, ou encore des pneus brûlés pour enrayer le système mondialisé du «couple véhicule-route» analysé par Yann Philippe Tastevin. Entre nature et culture, émotion et réflexion, matériel et idéel, nous habitons déjà le trouble, mais peut-être devrions-nous l'assumer, ou mieux, le semer.

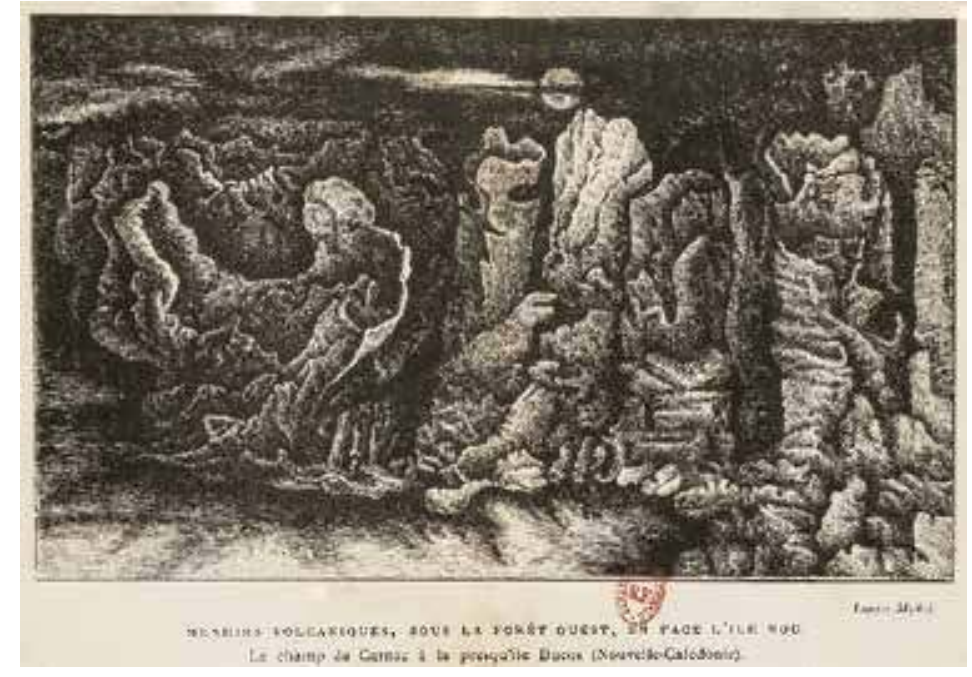

1. Menhirs volcaniques, sous la forêt ouest, en face de l'île Nou

Légendes et chants de gestes canaques. Avec dessins et vocabulaires, par Louise Michel (18301905), 1885

Les troubles, c'est aussi le nom du conflit sanglant qui s'est déployé en Irlande du Nord entre la fin des années 1960 et les années 2000, à la suite d'un soulèvement contre la ségrégation confessionnelle et les discriminations. Lalternance entre des épisodes meurtriers et des moments de paix relative bouleverse. La violence des insurgés clive. Parfois plus que la cause défendue, le choix des modes d'action, des armes et des instruments de la lutte divise, comme en témoigne également le mouvement des Droits civiques aux États-Unis, souvent représenté par deux figures, antagonistes et/ou complémentaires: celle de Martin Luther King, promoteur des 


\begin{abstract}
2. L'oppression nourrit la résistance. La résistance apporte la liberté.

Fresque réalisée sur un mur de la ville de Belfast (Divis Street) en mémoire de la bataille des Falls, quartier catholique ouvrier de Belfast où l'armée britannique, qui recherchait les armes des indépendantistes irlandais, imposa un couvre-feu et une occupation militaire de 36 heures en juillet 1970. Le couvre-

feu prit fin lorsque des centaines de femmes et d'enfants entrèrent dans la zone, munies de vivres pour les habitants. Durant l'événement, quatre civils irlandais furent tués, une soixantaine blessés et 337 arrêtés. L'armée britannique reconnut plus tard que certains de ses soldats commirent des pillages.
\end{abstract}

marches et des sermons pacifistes, et celle du partisan de l'autodéfense armée Malcom X. Si le premier a été consacré par l'édification d'un mémorial au sein du National Mall de Washington, l'autre a été condamné pour son discours The Ballot or the Bullet («le bulletin de vote ou la balle») du 3 avril 1964, au cours duquel il justifie le recours à la violence:

«Je ne me considère même pas comme un Américain. [...] Je suis l'une des vingt-deux millions de personnes noires qui sont les victimes de l'américanisme [...]. Il y aura des cocktails Molotov ce mois-ci, des grenades à main le mois prochain, et autre chose le mois suivant. [...] Ce sera la liberté, ou ce sera la mort.»

Il reprenait ainsi l'une des devises de la Révolution française, et avant elle, de la Guerre d'indépendance des États-Unis: «La liberté ou la mort ». Malcom X et Martin Luther King sont tous deux morts assassinés dans leur combat pour la liberté.

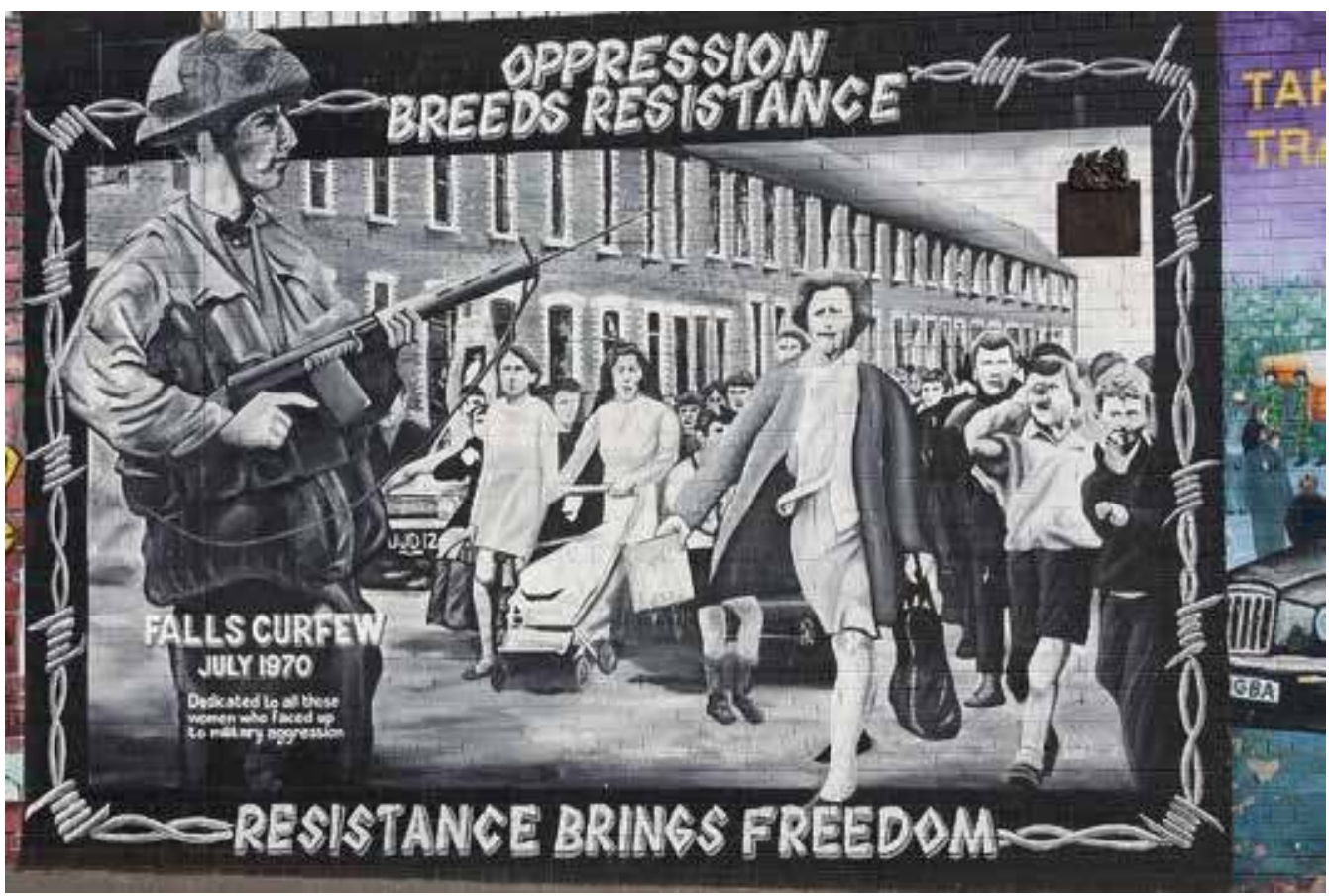

La violence est une notion polysémique largement instrumentalisée dans les représentations et les interprétations des luttes contemporaines. Si elle permet de mettre en lumière la conflictualité permanente autour de la définition des moyens de l'action, il arrive que, qualifiées de violentes, certaines luttes soient réduites à leurs gestes - les plus visibles - et que leur intelligence soit alors oubliée. Lors des «émeutes de banlieue» évoquées plus haut, la condamnation des émeutiers, «racailles» et autres «casseurs», a largement masqué les revendications sociales et politiques 
profondes de ce soulèvement populaire que Mohamed Mechmache, fondateur de l'association Aclefeu préfère qualifier de «révolte sociale». La disqualification de l'émeute, associée à la violence «de jeunes hommes issus de l'immigration postcoloniale» (Dorlin 2015: 35), a fait oublier les cahiers de doléances rédigés à l'époque et la diversité sociale et générationnelle des personnes révoltées (Bacqué 2015). Devenues moralement «intolérables» (Bourdelais \& Fassin 2005), les techniques violentes nous éloigneraient ainsi du pourquoi des luttes et orienteraient notre attention vers leur comment.

Si cette entrée par la violence permet de déplier progressivement le questionnement qui sous-tend ce numéro, elle nous alerte aussi sur les risques éthiques liés à un examen des manières d'agir en les disjoignant de la cause défendue - risques que soulève également, dans ce numéro, la contribution de Catherine Flood sur l'introduction au musée d'objets issus des mouvements sociaux. Attachés aux contextes de lutte, les modes opératoires se rappellent à eux. Ainsi l'exprime la plume déconstructiviste et réflexive de Bertolt Brecht dans le poème De la violence:

«On dit d'un fleuve emportant tout qu'il est violent. Mais on ne dit jamais rien de la violence des rives qui l'enserrent. » (Brecht 1967)

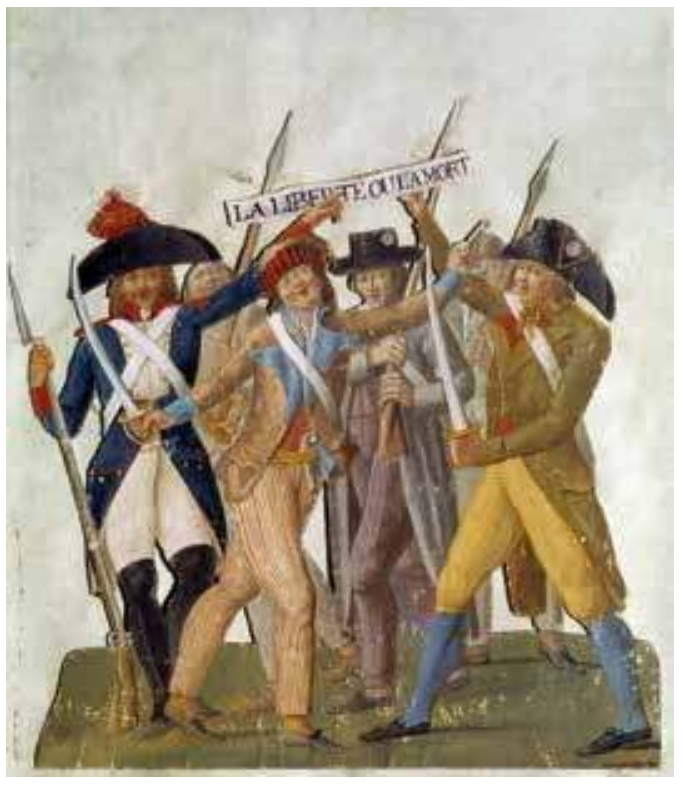

La violence est d'abord relationnelle, voire systémique, et les mots de Brecht révèlent la nature de ce système qui repose à la fois sur une qualification - est violent ce qui est qualifié comme «violent»- et une légitimation, soulevant ainsi la conflictualité qui existe autour de cette qualification. Qualifier les «violences policières» fait ainsi partie de la lutte des groupes souhaitant mettre cette question à l'agenda politique et médiatique, qu'ils parlent au nom des victimes d'interpellations mortelles dans les quartiers populaires ou bien des victimes d'affrontements avec les forces de l'ordre lors de manifestations ou au sein des Zones à défendre (ZAD). Ce numéro s'intéresse d'ailleurs aux techniques de soin que les blessures provoquées appellent: soin des corps dans l'urgence des manifestations (Perrine Poupin) et réparations collectives dans le temps plus long de la création d'un journal militant (Sébastien Galliot). Dans un État de droit, caractérisé par l'organisation d'un monopole de la violence physique légitime à travers des corps comme la police ou l'armée (Weber 1919), il est également important d'identifier d'autres formes de violences telles que la violence symbolique, intimement liée à la reproduction sociale (Bourdieu \& Passeron 1970). Une technique de lutte n'est en effet pas seulement un assemblage dynamique de moyens en vue de la contestation: elle est toujours aussi un point d'inflexion «minoritaire» et de retournement subversif de signification dans un contexte social «majoritaire» (Paveau 2019). En analysant la notion de bloc en politique, la contribution de Maxime Boidy montre ainsi la nécessité de penser ensemble les techniques du voir et les techniques de l'apparaître. La voie ouverte par la sociologie à une définition non physique de la violence permet par ailleurs de mettre en lumière le caractère violent des inégalités politiques, sociales,

3. Le cri français,
départ pour la frontière
d'un citoyen volontaire,
vers $1789 / 1794$
«Des citoyens de tous
état se rencontrant
dans les rues se réunis-
soient et poussoient
ensemble le terrible cris
de la Liberté ou la
Mort», Gouache de
Jean-Baptiste Lesueur.


économiques, environnementales, qui font grandir le sentiment d'injustice. Ces oppressions peuvent être le ferment de formes de confrontation originales de la part de celles et ceux qui les subissent, dans un renversement violemment symbolique, comme le montre la contribution de Magdalena Pérez Balbi sur l'insulte politique -escrache - envers des tortionnaires argentins.

À l'image de cette pratique qui pourrait faire l'objet de poursuites pour «injure publique et diffamation » ou pour «outrage», les techniques de lutte rassemblées dans ce numéro évoluent toujours sur une ligne de contestation, non pas seulement du pouvoir, mais aussi du droit, comme en témoignent le sabotage, c'est-à-dire la destruction de la propriété (François Jarrige); le vol, même temporaire, par exemple de chaises (Sandra Revolon); le trouble à l'ordre public, par la manifestation «non déclarée» (Violaine Chevrier), ou par la fabrication de barricades (Başak Ertür); ou encore le détournement de l'usage de la propriété publique, quand les blouses blanches sortent de l'hôpital (Thomas Billet, Leny Dourado \& Agnès Jeanjean). En effet, par-delà les fins et les causes défendues, la légitimation ou la sanction des moyens et des techniques de l'action est au cœur du rapport de force, y compris parmi les partisans d'une même cause. Outre la violence, les techniques de lutte recourant à l'humour, la joie, l'ironie ou encore le cynisme provoquent autant l'adhésion que la division. On le voit avec le détournement parodique et le «glam» des chorégraphies féministes présentées par Mikaëla Le Meur, ou encore la mise en scène de la mort du service public de l'enseignement supérieur de la recherche observée par Lucille Gallardo, avec la technique du die-in. Lutter, par tous les moyens, c'est finalement semer le trouble.

\section{Efficacité et matérialité des luttes}

Semer le trouble, c'est également produire une action efficace sur le monde social, à travers la mobilisation de ressources matérielles et la réalisation des gestes techniques, dont la diffusion ou la reproductibilité peuvent être une condition de réussite pour la lutte menée. Un manifeste qui appelle au ralliement d'un mot d'ordre ou une pétition qui soutient une protestation ont des modalités d'inscription dans le temps et dans l'espace qui conditionnent leur efficacité au sein du corps social tourmenté. Longtemps avant les stickers ou les affiches prêtes à coller (Violaine Chevrier; Élisabeth Lebovici), les post-it recouvrant les murs d'émotions politiques (Pierre-Olivier Dittmar), les kits numériques destinés à diffuser largement l'action désobéissante (Mikaëla Le Meur) ou encore les fiches techniques montrant comment fabriquer des masques contre les gaz lacrymogènes (Catherine Flood), l'invention alors récente de l'imprimerie joua un rôle déterminant dans la «provocation des placards». Au mois d'octobre 1534, ils couvrent par milliers les murs des grandes villes du royaume de François Ier, protestant contre la messe «papale» et invitant à la Réforme. L'historien Lucien Febvre décrit avec force détails combien la typographie adoptée sur les presses de Neuchâtel contribua au choc provoqué par cet affichage public clandestin, dont l'issue sera vingt-cinq ans plus tard les guerres de religion: 
«Typographie dense, mais bien disposée; titre en gros caractères gothiques faciles à lire pour ceux que déroutait encore le romain, pour ne point parler de l'italique; divisions bien apparentes: un court prologue, et quatre paragraphes parfaitement équilibrés par l'auteur, à la fois, et par un excellent typographe. De beaux blancs; des titres arithmétiques bien marqués [...] ; l'impression, à première vue, d'un texte très pesé, très calculé - d'une progression méthodique et rassurante dans le raisonnement. » (Febvre 1945: 62)

Au-delà des expériences singulières de la lutte, on constate cependant la récurrence statistique et la répétition de certains modes opératoires. S'ils nous semblent intimement liés à des configurations matérielles et des dispositifs techniques, c'est sur l'idée d'une limitation par le «stock des moyens d'action» dont disposeraient les groupes contestataires - un stock circonscrit à des figures et des routines caractéristiques de l'histoire sociale partagée - que Charles Tilly forme le concept de «répertoire d'action collective». Comme dans le jazz ou le théâtre, métaphores privilégiées par Tilly, un répertoire est un ensemble fermé de moyens qui forment une cohérence

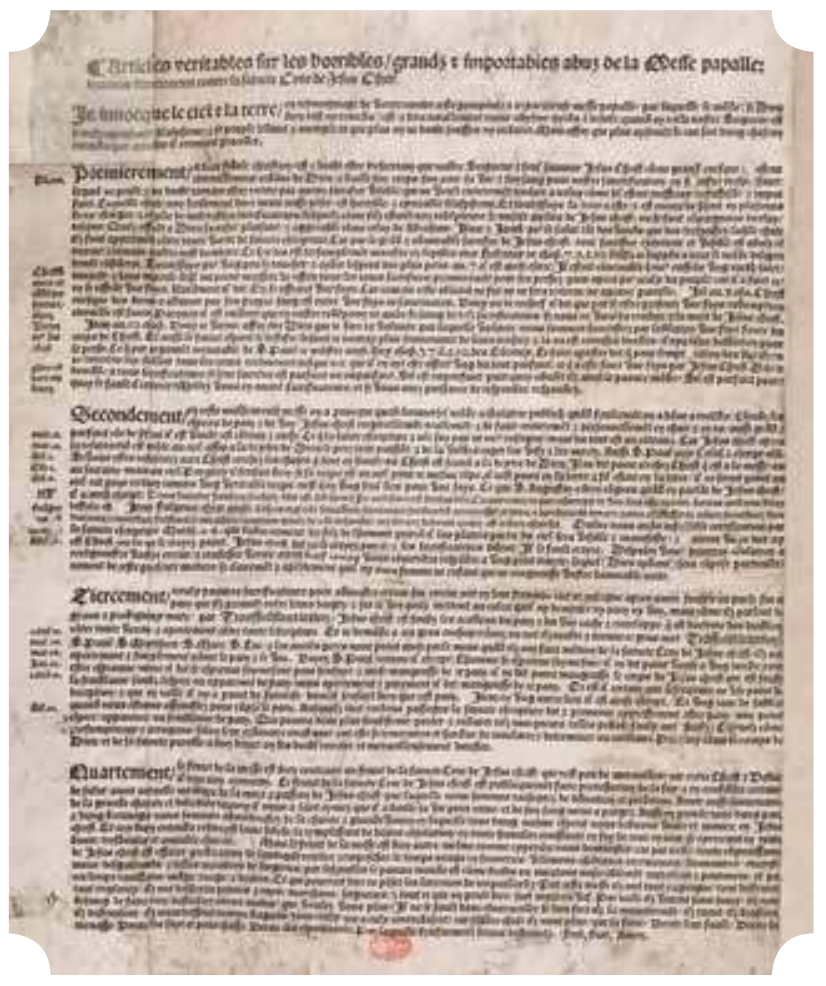
structurelle mais dont l'interprétation par des acteurs laisse place au choix, à l'invention et au développement d'un certain talent. Dans son dernier ouvrage, publié en 2008, Tilly prend soin de distinguer les actions ou tâches (marcher, clamer un slogan, entonner un chant), les performances (charivari, manifestation, piquet de grève) et les séquences (combinant plusieurs performances, tel qu'un die-in ou un déversement d'ordures qui clôt un cortège).

«Nous devons reconnaître que les répertoires de contestation sont des panoplies d'outils pour les personnes impliquées. Les outils servent plus d'une fin et leur efficacité relative dépend de la correspondance entre les outils, les tâches et les utilisateurs. Un nouveau répertoire est apparu au xixe siècle parce que de nouveaux utilisateurs se sont attelés à de nouvelles tâches et ont trouvé les outils disponibles inadaptés à leurs problèmes et à leurs compétences. Au cours des luttes actuelles, les personnes qui formulent des revendications et des contre-revendications ont créé de nouveaux moyens de revendiquer. Ils ont regroupé des performances complémentaires dans des répertoires. » (Tilly 2008: 64)

Certes, les contestataires interprètent des «scripts» au travers desquels ils innovent de façon généralement marginale et incrémentale - Mark Traugott (1995) fait à ce sujet une magistrale analyse de l'usage de la barricade dans les révoltes françaises d'Ancien Régime. Mais de nouvelles performances peuvent émerger lorsque les répercussions d'un mouvement de
4. Placard papale, 1534 contre la messe 
masse sur les institutions entraînent un changement d'environnement politique. Tilly mentionne notamment les années 1760, marquées par des mobilisations intenses en Grande Bretagne et dans les colonies américaines. Les «insurrections programmées», telles que les manifestations, avec la confection de banderoles et de bannières ou encore les affichages et pétitions qui s'imposent à l'orée du Xix ${ }^{e}$ siècle, supplanteraient le répertoire des émeutes du grain, des charivaris et des actions de rébellion contre les collecteurs de taxes. L'évolution diachronique des répertoires dépendrait ainsi du contexte politique structurel qui règle un «cycle de mobilisation», selon l'expression de Sidney Tarrow (Traugott 1995). Ce déterminisme du cadrage sociopolitique («l'environnement») induit une conception relativement statique et figée des sociétés. Le cortège de manifestation comme forme centrale de répertoire d'action à partir du XIXe siècle a par exemple entraîné la constitution d'un épais corps de doctrine du maintien de l'ordre et de diverses méthodes policières de contention et de répression, de sorte que les ajustements réciproques des forces en présence conduisent à une co-construction des techniques de lutte (Fillieule et al. 2016). On peut dès lors postuler, comme les tenants de la thèse du répertoire d'action et des cycles de mobilisation (Tilly \& Tarrow 2015), que l'environnement de contestation, c'est-à-dire les formes de gouvernementalité et leurs dispositifs de sécurité (Foucault 2004 [1978]) ont une fonction décisive dans le choix possible des outils et le surgissement d'éventuelles modifications de répertoire. Cette conception présente selon nous plusieurs défauts.

Associer actions et performances contestataires au sein d'un répertoire incline non seulement à minorer leur potentiel de changement social, mais le répertoire d'action tend encore à ne distinguer que les modes de contestation visibles, hétérogènes et saillants. Le site internet animé par le chercheur Alain Bertho (Anthropologie du présent) offre ainsi une collection d'un seul et unique «répertoire contemporain de la révolte» fondé sur le nombre et sous-tendu par l'existence d'une masse critique, dont la répétition statistique permet de créer diagrammes et cartes (Bertho 2009). En dehors de ce répertoire, que deviennent les actions non ouvertes, les résistances de l'ombre, les formes contestataires érigées en modes de vie (tels que les squats artistiques ou militants)? Ou encore les postures de lutte minoritaires, voire individuelles, qui sont pour certaines «silencieuses» et non adossées à un régime de publicisation du désaccord?

Élargissant un terrain consacré aux paysans malais, l'anthropologue James C. Scott a justement proposé une caractérisation des actes de résistance diffus, continus et «infrapolitiques » au travers ces ruses ourdies vis-à-vis de la domination. L'opposition qu'il construit entre un «texte public attestant une soumission passive et un «texte caché » impliquant au contraire défiances et «mauvais esprit», restitue aux subalternes une agentivité certaine (Scott 2009). Des réseaux informels et discrets composent ainsi les «arts de la résistance», qui sont d'un registre tant matériel que symbolique (voir par exemple Georges Favraud sur les stratégies taoïstes de la lutte dans ce numéro). Approcher les pratiques dissidentes par l'investissement de sens que leur reconnaissent leurs protagonistes met l'accent sur les «tactiques» (Certeau 1990) de détournement ou de contournement symbolique en tant que productrices d'émancipation, au risque toutefois de privilégier une perspective simplement «consolatrice» (Bayat 2013). 
Parce qu'une technique n'est pas neutre en termes d'intentionnalité et de régime de fonctionnement, l'étude de la culture matérielle ne peut ignorer les objets « désobéissants (Flood \& Grindon 2014) pour leur capacité critique à altérer ou contrecarrer, pour ceux qui les façonnent, le déterminisme induit par un design univoque. Là où l'historiographie du répertoire d'action incite à limiter le «stock» des moyens de lutte et à en fermer le répertoire, la technologie culturelle s'intéresse au contraire au jeu et à l'ouverture qui caractérisent le registre matériel des mobilisations. Les études rassemblées dans ce volume en montrent la diversité: par exemple, le potentiel de délinquance et de subversion logé dans les routines numériques (Jean-Paul Fourmentraux; Nicolas Nova et Félicien Goguey), l'enchevêtrement du politique et de l'écologique au travers d'une chorégraphie (Joanne Clavel), ou encore les bifurcations et parfois étranges chemins empruntés par la lutte pour l'autonomie, via la défense politique et esthétique de zones convoitées (Raphaële Bertho $\&$ Jürgen Nefzger), la résistance et le détournement électrique (Umberto Cao) ou encore les alliances avec les arbres (Lucie Dupré). Et bien que la valeur de "créativité » infuse dans l'ensemble du dernier capitalisme (Boltanski \& Chiapello 1999) et dans les techniques de maniement des hommes (Le Texier 2016), les «arts de désobéir» ne sont pas en reste d'invention et de construction de nouveaux styles de vie, dans lesquels les artistes activistes subvertissent les normes et les canons esthétiques (Thomas Golsenne; Élisabeth Lebovici). Il est vrai qu'obéir comme désobéir, «c'est donner forme [nous soulignons] à sa liberté » (Gros 2017: 40). Paraphrasant Carl von Clausewitz, Bryan Pfaffenberger (1992) écrit quant à lui que «la technologie n'est pas la politique continuée par d'autres moyens, c'est la politique construite par des moyens technologiques». La politisation de la réalité matérielle se loge ainsi jusqu'au cœur des chaînes opératoires, comme en témoignent les «objets de grève », qui «emboîtent» la contestation dans la chaîne de production de la marchandise. C'est ce que nous voyons avec la montre Lip dont le boîtier indique «vente sauvage» (1974) ou bien les flacons du «parfum de solidarité » diffusés par les ouvriers de l'usine Bourgogne Application Plastique pour financer leur mouvement de résistance à la fermeture du site (1996). Ces exemples nous invitent à considérer davantage la

5. Parfums de solidarité,
1996
Les ouvriers de l'usine
Bourgogne Application
Plastique, en Côte d'Or,
vendirent plusieurs mil-
liers de flacons en plas-
tique moulé du «Parfum
de solidarité » afin de
financer leur grève illimi-
tée avec occupation des
locaux. Cet « objet de
grève» fut collecté, ainsi
que 39 autres, parl'artiste
lean-Luc Moulène.

5. Parfums de solidarité, Les ouvriers de l'usine Bourgogne Application vendirent plusieurs milliers de flacons en plasde solidarité $»$ fin de financer leur grève illimiavec occupation des que 39 autres, par l'artiste Jean-Luc Moulène. 
résistance des choses, des objets politiques qui «forcent la pensée» (Stengers 2010) en même temps qu'ils soutiennent et donnent corps à des aspirations et des combats.

\section{Outiller l'avenir}

L'idéalisme critique et l'imagination révolutionnaire, qu'ils soient (dis)qualifiés de romantiques ou non, sont des moteurs puissants de la lutte permanente contre le «désenchantement du monde» (Löwy 2003), qui trouve son expression contemporaine dans la maxime TINA « There is no alternative »: il n'y a pas d'alternative. Des alternatives, il en existe pourtant, comme en témoigne la multiplication des collectifs néo-paysans qui « retournent » à la terre en se réappropriant outils et techniques agricoles dans une démarche autonome, imaginative et « conviviale » (Madeleine Sallustio). Elles sont en germe dans les brèches du pouvoir, dans l'intimité des foyers comme par exemple en Mongolie, où l'on « compose » pour résister, pour reprendre les mots de Sandrine Ruhlmann. Elles naissent également de l'échange et de la prise de conscience de « ce à quoi nous tenons» (Hache 2011) via l'usage de techniques d'auto-description (Consortium Où atterrir?) et de cartographie des espaces en danger, qui requièrent une protection particulière, comme dans la lutte contre les logements insalubres marseillais que traite Zoé Carle. C'est ce que montrent également Caroline Desbiens et Irène Hirt à travers les luttes autochtones amérindiennes, qui cherchent à exister sur la mappemonde. D'autres espaces alternatifs se créent dans les cercles aborigènes où les techniques de parole thérapeutiques mobilisent des objets capables de régénérer les forces: échanger ses maux, selon Edgar Tasia, serait un premier pas vers une résistance contre la domination.

Si la lutte est faite d'une multitude de petites choses, de petits réenchantements, de petites victoires, reviendrait-elle à l'art et la manière d'«avoir l'histoire à l'usure» (Dorlin 2017: 150) ? Quitte à, en parallèle, se préparer à l'effondrement, au désastre ou à s'accommoder d'une vie dans les ruines, nécessitant le recours à des expédients écologiques d'adaptation (Tsing 2017)? Si cette poétique du précaire et de la fragilité résiliente est séduisante, gardons-nous de la dresser en paradigme et de servir un discours dominant nous sommant de nous adapter (Stiegler 2019). En miroir de cette recherche d'harmonie, d'ajustement durable produit par une multiplicité de résistances, s'agirait-il au contraire de se défendre et de s'opposer «quoiqu'il en coûte à la domination, au risque de provoquer l'affrontement?

Comment résister? Ou bien plutôt : comment «juste rester en vie» pour paraphraser Rodney King durant le procès des policiers qui l'avaient lynché à Los Angeles en 1991 et qui furent acquittés (Dorlin 2017). Tel est l'enjeu de ce numéro sur les objets et les techniques de lutte. Il convoque le génie subversif des mille et une façons de contester, par la matière, les objets, les corps, les muscles. La perspective est néanmoins réparatrice, tant nous semble évidente «la nécessité de guérir ensemble» (Stengers 2019: 19). Après avoir détaillé les manières avec lesquelles les cœurs peuvent se soulever-Première partie: Voies du soulèvement-nous sillonnerons 


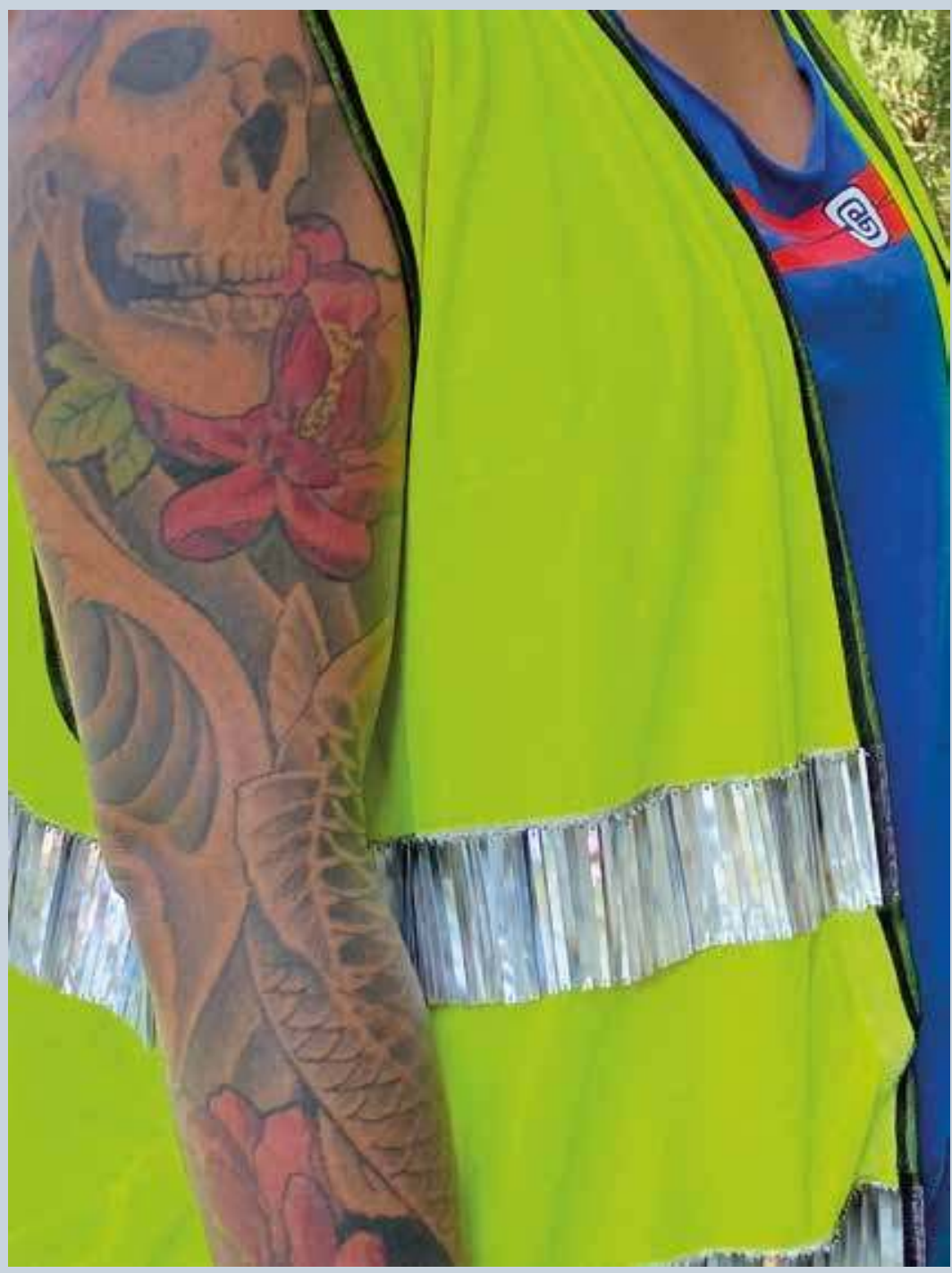

\section{CEuvre de Jean-Daniel Devatine, 2019}

«L'une des tenues les plus spectaculaires de Tahiti collectée lors de la seconde moitié du xviIIe siècle par les Anglais est l'habit du deuilleur, utilisé lors de processions funéraires. Selon les récits de témoins, les personnes se trouvant sur le parcours de la procession du maitre de cérémonie, le deuilleur, étaient battues par d'autres à l'aide de bâtons. Cet habit de deuil, objet de la procession, était composé de plusieurs éléments d'un extrême raffinement. II comportait notamment un plastron de milliers de fines lamelles de nacres grises réfléchissantes, attachées les unes aux autres. Les rassemblements hebdomadaires de Gilets jaunes au cours de l'année 2019 en France, mais aussi dans plusieurs pays d'Europe et dans le monde, m'ont amené à repenser les réflecteurs de ces chasubles de sécurité, signe du ralliement au mouvement. C'est ainsi que des nacres grises similaires à celles de l'habit du deuilleur ont habillé les bandes réfléchissantes du gilet jaune. Lors d'épisodes de violente répression du mouvement des Gilets jaunes, les matraquages, tirs de flash-balls et autres grenades furent largement le lot des manifestants. » 
les chemins de traverse de la lutte, dans ses détournements et ses retournements-Deuxième partie: Arts de la subversion - pour conclure notre voyage à travers les révoltes d'hier et d'aujourd'hui en retrouvant réconfort et convivialité - Troisième partie: Refuges et pratiques réparatrices - et en remplissant nos estomacs (voir les subsistances de Florent Grouazel). Ainsi alimentés, équipés et renforcés, nous repartirons battre le pavé.

\section{Iconographie}

Image d'ouverture. I can't breathe. Dans le sillage d'une manifestation consécutive à la mort de George Floyd, afro-américain asphyxié un genou sur la nuque dans le cadre d'une arrestation de police le 25 mai 2020. Photo prise à Grand Army Plaza, dans l'arrondissement de Brooklyn à New York, le 7 juin 2020. (c) Rhododendrites.

1. Bibliothèque nationale de France.

2. (C) Ardfern, 2 mai 2011.
3. Bibliothèque nationale de France.

4. Bibliothèque nationale de France, réserve des livres rares, D2-453.

5. Archives nationales du monde du travail (Roubaix), photographie de Mélody Fernandez, Fonds Jean-Luc Moulène (cote 2003 025).

6. (C) Jean-Daniel Devatine.

\section{Les auteurs}

Matthieu Duperrex est maître de conférences en sciences humaines à l'École nationale supérieure d'architecture de Marseille. Chercheur à l'Inama et directeur artistique du collectif Urbain, trop urbain (www.urbain-trop-urbain.fr), ses travaux s'inscrivent dans le champ des humanités environnementales.

Mikaëla Le Meur est docteure en anthropologie. Actuellement enseignante en sociologie et en science politique à Aix-Marseille Université, ses recherches au Vietnam et en France portent notamment sur l'écologie et la matérialité du politique.

\section{Références}

Bacqué, M.-H. 2015 «Les révoltes de 2005, une prise de conscience politique. Entretien avec Mohamed Mechmache», Mouvements 3(83): 17-21.

Bayat, A. 2013 Life As Politics. How Ordinary People Change the Middle East. Stanford: Stanford University Press.

Bertho, Al. 2009 Le temps des émeutes. Paris: Bayard.

Boltanski, L. \& E. Chiapello 1999 Le nouvel esprit du capitalisme. Paris: Gallimard.

Bourdelais, P. \& D. Fassin dir. 2005 Les constructions de l'intolérable. Études d'anthropologie et d'histoire sur les frontières de l'espace moral. Recherches. Paris: La Découverte.

Bourdieu, P. \& J.-C. Passeron 1970 La reproduction. Paris: Éditions de Minuit.
Braun, B. \& S. Whatmore dir. 2010 Political Matter Technoscience, Democracy, and Public Life. Minneapolis: University of Minnesota Press.

Brecht, B. 1967 Poèmes. Tome 5. Paris: LArche.

Butler, J. 2006 Trouble dans le genre. Le féminisme et la subversion de l'identité. Trad. Cynthia Kraus. Paris: La Découverte.

Caeymaex, F., Despret, V. \& J. Pieron dir. 2019 Habiter le trouble avec Donna Haraway. Bellevaux: Éditions Dehors.

Certeau, M. de 1990 L'invention du quotidien. 1. Arts de faire. Paris: Gallimard.

Dorlin, E. 2015 «Le cœur de la révolte», Mouvements $3(83): 35-41$.

Dorlin, E. 2017 Se défendre. Une philosophie de la violence. Paris: La Découverte. 
Febvre, L. 1945 "L'origine des placards de 1534 », Bibliothèque d'Humanisme et Renaissance 7: 62-75.

Fillieule, O., Viot, P. \& G. Descloux 2016 «Vers un modèle européen de gestion policière des foules protestataires? », Revue française de science politique 66 (2): 295-310.

Flood, C. \& G. Grindon dir. 2014 Disobedient Objects. Londres: V\&A Publishing.

Foucault, M. 2004 [1978] Sécurité, territoire, population. Cours au Collège de France, 1977-1978. Paris: GallimardSeuil (Hautes études).

Gros, F. 2017 Désobéir. Paris: Albin Michel.

Hache, E. 2011 Ce à quoi nous tenons. Propositions pour une écologie pragmatique. Paris: La Découverte.

Haraway, D. J. 2020 Vivre avec le trouble. Trad. Vivien Garcia. Vaulx-en-Velin: Les Éditions des Mondes à faire.

Le Texier, T. 2016 Le maniement des hommes. Essai sur la rationalité managériale. Paris: La Découverte.

Löwy, M. 2003 « Gustav Landauer, révolutionnaire romantique», Tumultes 1 (20): 93-103.

Malm, A. 2020 Comment saboter un pipeline. Trad. Étienne Dobenesque. Paris: La Fabrique.

Milton, K. 2002 Loving Nature. Towards an Ecology of Emotion. Londres: Routledge.

Paveau, M.-A. 2019 «La resignification. Pratiques technodiscursives de répétition subversive sur le web relationnel », Langage et société 2 (167): 111-141.

Perrot, M. 2001 Les ouvriers en grève. France 1871-1890. 3 volumes. Les ré-impressions. Paris: Éditions de l'EHESS.

Pfaffenberger, B. 1992 «Technological Dramas ». Science, Technology, \& Human Values 17 (3): 282-312.
Rancière, J. 2000 Le partage du sensible. Esthétique et politique. Paris: La Fabrique.

Scott, J. C. 2009 La domination et les arts de la résistance. Fragments du discours subalterne. Trad. Olivier Ruchet. Paris: Éditions Amsterdam.

Stengers, I. 2010 «Including non-humans in political theory. Opening Pandora's box? » in B. Braun \& S. Whatmore dir. Political Matter. Technoscience, Democracy, and Public Life. Minneapolis: University of Minnesota Press: 3-33.

Stengers, I. 2019 Résister au désastre. Marseille: Éditions Wildproject.

Stiegler, B. 2019 «Il faut s'adapter ». Sur un nouvel impératif politique. Paris: Gallimard.

Tchernookov, J. 2018 «Louise Michel, une femme libre au bagne" La Bibliothèque numérique de la BnF et de ses partenaires. Le Blog Gallica (blog) 20/08/2018. [En ligne] : gallica.bnf.fr/blog/20082018/ louise-michel-une-femme-libre-au-bagne.

Tilly, C. 2008 Contentious Performances. Cambridge: Cambridge University Press.

Tilly, C. \& S. Tarrow 2015 Politique(s) du conflit. De la grève à la révolution. Trad. Rachel Bouyssou. Paris: Presses de Sciences Po.

Traïni, C. dir. 2009 Émotions... Mobilisation! Paris: Presses de Sciences Po

Traugott, M. dir. 1995 Repertoires and Cycles of Collective Action. Durham: Duke University Press.

Tsing, A. L. 2017 Le champignon de la fin du monde. Sur la possibilité de vivre dans les ruines du capitalisme. Trad. Philippe Pignarre. Paris: La Découverte (Les Empêcheurs de penser en rond).

Weber, M. 2003 [1919] Le savant et le politique. Trad. Catherine Colliot-Thélène. Paris: La Découverte.

\section{Remerciements}

Matthieu Duperrex remercie la Fondation Jan Michalski pour son accueil lors de la préparation estivale de ce numéro, qu'il dédie à la mémoire de Chéryl Amade-Duperrex, combattante de la Terre Mère.

\section{Pour citer l'article}

Duperrex, M. \& M. Le Meur 2020 «Matières à friction et techniques de lutte», TechniquesE Culture 74 «Semer le trouble. Soulèvements, subversions, refuges», p. 12-25. 